\title{
ANALYSIS OF FILTERING MATERIAL AND ITS EFFECT ON X-RAY FEATURES BY USING COMPUTATIONAL METHOD FOR MEDICAL IMAGING APPLICATIONS
}

\begin{abstract}
Hüseyin Ozan Tekin'* ${ }^{*}$ Ümit Kara²
${ }^{1}$ Üsküdar University, Vocational School of Health Services, Radiotherapy Department, İstanbul, Turkey ${ }^{2}$ Süleyman Demirel University, Vocational School of Health Services, Medical Imaging Department, Isparta, Turkey

Abstract. In recent years, $X$-rays with different radiological devices have been frequently used. $X$-rays are produced in vacuum tubes by bombarding a metal target with high-speed electrons whereas radiological images occur after the resulting radiation passes through the patient's body onto a photographic plate or a digital recorder to produce a radiological image. In radiology, devices that use X-rays can be classified as radiography, fluoroscopy, mammography and CT. In this study, we present a general approach for the simulation of X-ray spectra emitted from targets bombarded with electron beams for different energy ranges which are widely used in different radiological devices. The electron and photon transport is simulated by using the SpekCalc GUI code (version 1.1). In this study, we calculated the total photon spectra of the Tungsten (W) target for $150 \mathrm{keV}$ and $12^{\mathrm{c}}$ anode angle and certain energies for required energy values which are necessary for a successful radiodiagnostic process. We also performed simulations with the same parameters by adding the filter material into the X-ray tube. We achieved a good agreement between the clinical data and the computational method. The results showed that the computational method is in accordance with the manufacturer parameters.
\end{abstract}

Key words: X-ray filtration, SpekCalc GUI, medical imaging

DOI: $10.21175 / \operatorname{RadProc.2016.31}$

\section{INTRODUCTION}

Medical imaging is a technique that is usually known as visual representation of the parts of the body for clinical studies and analysis. Imaging process for medical aims is performed by staff which includes radiologist, radiographers/radiology technicians, sonographers, engineers, medical physicist and nurses. The harmony between staff members is seriously important for a successful radiodiagnostic process. Of course, imaging devices also have an important place in the quality of the medical imaging which is also significant for the radiation justification process. Having in mind the development of technology, we can basically classify the medical imaging devices as PET/CT/OCT, digital x-ray, ultrasound, MRI, hyperspectral imaging 3D/4D imagers, vein viewer [1]. As a separate issue from the medical imaging devices, we can also mention X-ray devices such as radiography, computed tomography (CT), dental conebeam computed tomography, fluoroscopy and mammography. What these devices have in common is that they use X-rays during the medical imaging process. An x-ray beam is generated in a vacuum tube which is mainly composed of anode and cathode materials to produce an X-ray beam. Anode represents the component in which the X-ray beam is produced and it is made from a piece of metal. Nowadays, most $\mathrm{X}$-ray tubes are built of Tungsten $(\mathrm{Z}=74)$ with its high atomic number and high melting point of $3370^{\circ} \mathrm{C}$ with the low rate of volatilization. Figure 1 represents the cross-sectional view of an x-ray tube and the anode position inside of the tube.

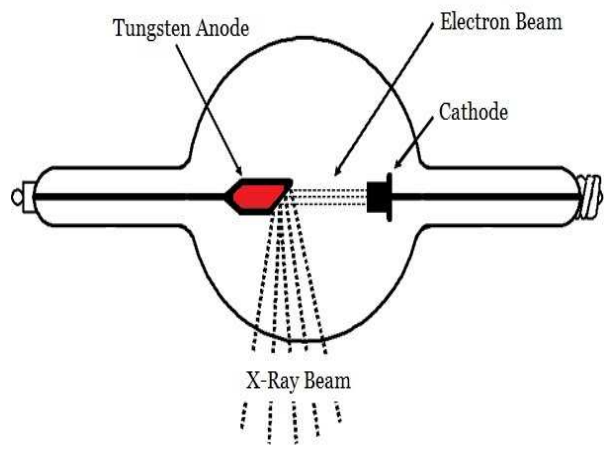

Figure 1. Schematic view of $x$-ray tube and position of anode

Since the anode material is located under a certain angle in the tube, some special conditions can occur, such as the heel effect. For decades, the heel effect has been known to cause an angular dependence of the emitted spectrum of an x-ray tube [2]. The knowledge about the heel effect in radiological devices is the best way to get images of a patient for the diagnosis. The clinical model and parameters was validated by comparing the computational calculated photon beam

\footnotetext{
*huseyinozan.tekin@uskudar.edu.tr
} 
spectra with the results of SpekCalc software (version 1.1). If the optimum $\mathrm{keV}$ value as electron energy and best anode angle are used, it provides the opportunity for a successful diagnostic process. Of course, there is no fixed value for different devices. Each device may use different values and parameters associated with the device structure and purpose. However, a common feature of different parameters in different devices is to supply a high quality $\mathrm{x}$-ray beam and to direct onto patient. For this aim, we considered some different devices' manufacturer parameters.

\section{MATERIALS AND METHODS}

In the current study, we considered manufacturers' parameters for calculations. We chose mammography, radiography, angiography and fluoroscopy as a case study of the subject. Tungsten (W) is the most widely used anode material in devices. Mammography $\mathrm{x}$-ray tubes use a rotating anode inside. Molybdenum (Mo) is the most frequently used anode material for obtaining the best energy peaks for imaging of a breast. We considered the parameters shown in Table 1 as the manufacturer parameters of Siemens OEM Products [3].

Table 1. Parameters of Siemens OEM Products

\begin{tabular}{|l|c|c|l|}
\hline \multicolumn{1}{|c|}{ X-Ray Tube } & $\begin{array}{c}\text { Nominal } \\
\text { Voltage }\end{array}$ & $\begin{array}{c}\text { Anode } \\
\text { Angle }\end{array}$ & Application \\
\hline P 135/30 R & $135 \mathrm{keV}$ & $15^{\circ}$ & $\begin{array}{l}\text { Radiography } \\
\text { Fluoroscopy }\end{array}$ \\
\hline RAY-8 & $150 \mathrm{keV}$ & $16^{\circ}$ & $\begin{array}{l}\text { Radiography } \\
\text { Fluoroscopy }\end{array}$ \\
\hline RAY-12 & $150 \mathrm{keV}$ & $12^{\circ}$ & $\begin{array}{l}\text { Radiography } \\
\text { Fluoroscopy }\end{array}$ \\
\hline RAY-14 & $150 \mathrm{keV}$ & $12^{\circ}$ & $\begin{array}{l}\text { Radiography } \\
\text { Fluoroscopy }\end{array}$ \\
\hline $\begin{array}{l}\text { Optilix } \\
154 / 30 / 50 \mathrm{R}\end{array}$ & $150 \mathrm{keV}$ & $16^{\circ}$ & $\begin{array}{l}\text { Radiography } \\
\text { Fluoroscopy }\end{array}$ \\
\hline SV 150/30/50 C & $150 \mathrm{keV}$ & $16^{\circ}$ & $\begin{array}{l}\text { Radiography } \\
\text { Fluoroscopy }\end{array}$ \\
\hline SV150/40/80 C & $150 \mathrm{keV}$ & $12^{\circ}$ & $\begin{array}{l}\text { Radiography } \\
\text { Fluoroscopy } \\
\text { Angiography }\end{array}$ \\
\hline P40MoW-100G & $40 \mathrm{keV}$ & $20^{\circ}$ & Mammography \\
\hline
\end{tabular}

Radiological device parameters used by the manufacturer are defined in detail and input in SpekCalc program. A software program, SpekCalc, is used for the calculation of $x$-ray spectra from tungsten anode $\mathrm{x}$-ray tubes. SpekCalc is primarily designed to be used in medical physics for both research and education aims. Noteworthy is the particularly wide range of tube potentials $(40-300 \mathrm{keV})$ and anode angles (recommended: 6-30 degrees) that can be modeled: the program can therefore be potentially useful to those working in superficial/orthovoltage radiotherapy, as well as in diagnostic radiology [4].

\section{RESULTS}

The optimum values of nominal voltage and anode angle are seriously important for the best medical image of a patient. It is extremely important to obtain a quality medical image not only to analyze the image in order to perform diagnostic procedures but also to, at the same time, prevent the patient from receiving the radiation again. In Figure 2, we can see the simple schematic view of the simulation model. As we see from Figure 2, there is the anode angle $\theta$ according upstream of the electron beam and between $\mathrm{c}$ and $\mathrm{d}$ point effective focal spot size of $x$-ray beam were optimum useful x-ray used for diagnostic process.

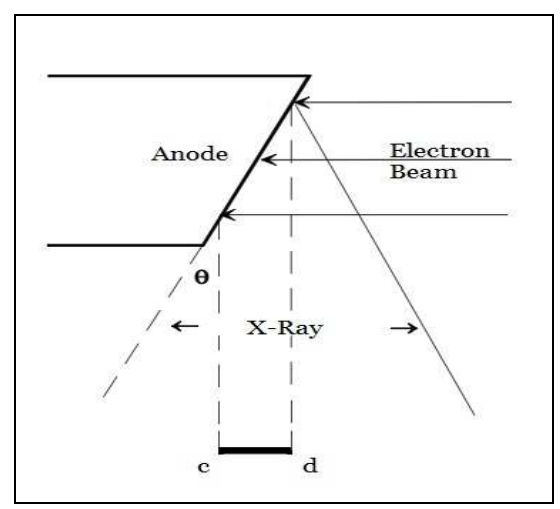

Figure 2. Schematic view of simulation process

In this study, we choose the Siemens SV150/40/80 C model which can be used for radiography, fluoroscopy and angiography clinical examinations for our simulations. As the manufacturer uses a nominal voltage value and anode angle, we calculated the x-ray intensities to see the validation of the Computational simulation by comparing the manufacturer's parameters. The first calculation has been done by using the values of a nominal voltage of $150 \mathrm{keV}$ and the anode angle of $12^{\circ}$ respectively and without choosing any filter materials. We obtained the x-ray spectra shown in Figure 3 and pointed out the peak value at $59.3 \mathrm{keV}$. Here, we can see the maximum peak intensity at the mentioned peak value.

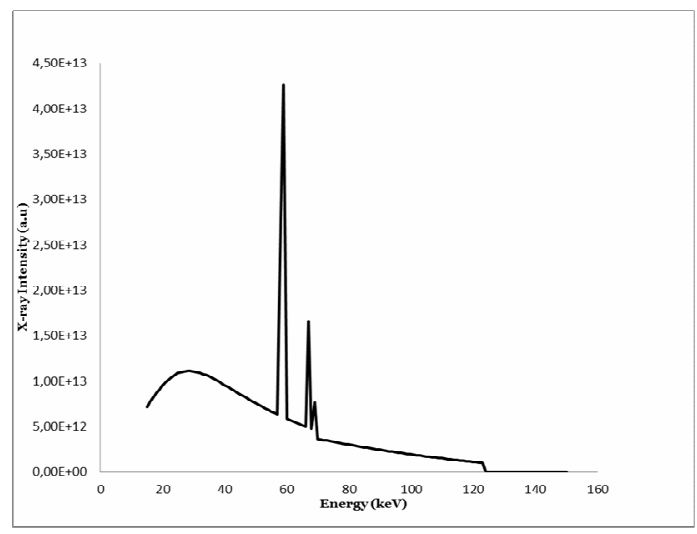

Figure 3. X-ray spectra of Tungsten $\left(150 \mathrm{keV}-12^{\circ}\right)$

Also, we can see some other peaks with different energies which are not useful for diagnostic procedures. This situation is, of course, undesirable for the patient regarding the absorbed dose in tissue during the examination. To prevent it, we used filtration and obtained Figure 4. Aluminum is the most commonly added filtration material. Copper can also be used for improving low-energy x-ray filtering [5]. Figure 4 was obtained by adding the $2.5 \mathrm{~mm}$ Al filter 
into the simulation and by considering manufacturer parameters. We used $2.5 \mathrm{~mm}$ total filtration and $\mathrm{Al}$ equivalent in the tube assembly [6].

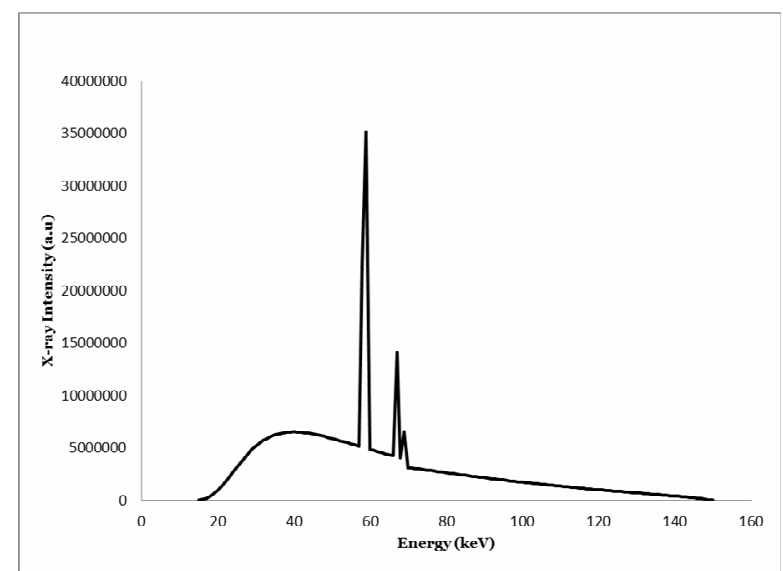

Figure 4. X-ray spectra of Tungsten $\left(150 \mathrm{keV}-12^{\circ}-2.5 \mathrm{Al}\right.$ Filter)

As we see from Figure 4, there is a significant decrease in useless x-rays and a significant increase in useful $x$-rays for the medical imaging process. This process is a factor that reduces the unnecessary dose rate received by the patient and allows better examination.

\section{CONCLUSION}

In recent years, studies on medical imaging have indicated that the use of a smaller dose rate and the application of optimum imaging parameters are extremely important for a successful imaging process and the patient's health. In this study, we investigated the validation of the Computational method for such scientific application. It can be concluded that the Computational method is a strong tool and consistent with the clinical device parameters. Our results showed that the parameters used by the manufacturer are ideal and optimal for a successful medical imaging process. It can be also concluded that the Computational method can be used for future studies on device optimization and for approaching new technologies.

\section{REFERENCES}

1. $\mathrm{X} . \mathrm{Xu}$ and E. Bartolome, Introduction to Medical Imaging, Medical Business Unit, Texas Instruments, Dallas (TX), USA, 2010

2. H. Braun, Y. Kyriakou, M. Kachelrieß and W.A. Kalender, "The Influence of the Heel Effect in ConeBeam Computed Tomography: Artifacts in Standard and Novel Geometries and Their Correction," Phys. Med. Biol., vol. 55, no. 19, Sep. 2010

3. Siemens OEM Products, Siemens, Erlangen, Germany Retrieved from: www.siemens.com/oemproducts; Retrieved on: 18.03.2016

4. G. Poludniowski, G. Landry, F. De Blois, P.M. Evans and F. Verhaegen, "SpekCalc: A Program to Calculate Photon Spectra from Tungsten Anode X-Ray Tube," Phys. Med. Biol., vol. 54, no. 19, pp. N433-N438, 2009

5. K.J. Strauss, "Cardiac Catheterization Equipment Requirements: Pediatric Catheterization Laboratory Considerations," in Syllabus: a Categorical Course in Diagnostic Radiology Physics-Cardiac Catheterization Imaging, E.L. Nickoloff, K.J. Strauss, Eds., Oak Brook (IL), USA: RSNA, 1998, pp. 105-119

6. Device Parameters of SV $150 / 40 / 80$ C 100 L, IRS Technology, Italy

Retrieved from: http://www.irstechnology.it/files/SV150-40-80-.pdf;

Retrieved on: 18.03.2016 